\title{
High-intensity terahertz radiation from a microstructured large-area photoconductor
}

\author{
A. Dreyhaupt, S. Winnerl, T. Dekorsy, and M. Helm \\ Forschungszentrum Rossendorf, Institute of Ion Beam Physics and Materials Research, \\ POB 5101 19, 01314 Dresden, Germany
}

\begin{abstract}
We present a planar large-area photoconducting emitter for impulsive generation of terahertz $(\mathrm{THz})$ radiation. The device consists of an interdigitated electrode metal-semiconductor-metal (MSM) structure which is masked by a second metallization layer isolated from the MSM electrodes. The second layer blocks optical excitation in every second period of the MSM finger structure. Hence charge carriers are excited only in those periods of the MSM structure which exhibit a unidirectional electric field. Constructive interference of the $\mathrm{THz}$ emission from accelerated carriers leads to $\mathrm{THz}$ electric field amplitudes up to $85 \mathrm{~V} / \mathrm{cm}$ when excited with fs optical pulses from a Ti:sapphire oscillator with an average power of $100 \mathrm{~mW}$ at a bias voltage of $65 \mathrm{~V}$ applied to the MSM structure. The proposed device structure has a large potential for large-area high-power THz emitters.
\end{abstract}

The development of new terahertz $(\mathrm{THz})$ sources is of great importance for a large variety of scientific and technological applications. For the generation of $\mathrm{THz}$ waves with fs pulses several emitter concepts have been employed like optical rectification, ${ }^{1}$ phase-matched difference-frequency mixing, ${ }^{2}$ surface field $\mathrm{THz}$ emitters, ${ }^{3}$ photoconductive switches, ${ }^{4,5}$ or biased pin-diodes. ${ }^{6}$ Several attempts have been made to increase the $\mathrm{THz}$ emission efficiency by employing photoconductors with laterally structured emitter electrodes. ${ }^{7,8}$ The driving forces for further developments of impulsive $\mathrm{THz}$ emitters are applications which require a large bandwidth and/or high $\mathrm{THz}$ electric field amplitudes. The performance of $\mathrm{THz}$ emitters based on photoconductors (PC) is limited by several conditions. In order to achieve a high bandwidth the electric field applied to the PC should be high in the $100 \mathrm{kV} / \mathrm{cm}$ range, i.e., close to the breakdown voltage of the PC. On the other hand the optically excited area should be large to prevent local heating of the PC with the exciting laser pulses which would have a detrimental effect on the carrier mobility and thus decrease the bandwidth. Therefore the solution of using pin-diodes has advantages over lateral electrical contacts to the PC, since in a pin-diode high electric fields exist in the whole area of the diode. Yet the acceleration of carriers in a pin-diode is perpendicular to the surface which results in a bad outcoupling efficiency of the dipole radiation with the main intensity being emitted parallel to the surface. Hence the acceleration of carriers in the plane of the PC would be favorable. However, large lateral electric fields applied over large areas, i.e., large electrode spacing, require pulsed high voltage sources in the $\mathrm{kV}$ range which one tries to avoid because of the electronic interference with sensitive electronic equipment in the laboratory. ${ }^{9}$ Another limitation of PC emitters with lateral carrier excitation is the emission of the radiation via coupling to a microstructured dipole antenna, which typically exhibits a narrower bandwidth than the intrinsic carrier acceleration in the PC.

Here we investigate the $\mathrm{THz}$ radiation from photoconductive MSM structures modified in a way to achieve unidirectional carrier acceleration on a large active area for high excitation powers. The emission is based on the intrinsic acceleration of carriers in the PC and hence does not require a narrow-band antenna. High electric fields of the order of $100 \mathrm{kV} / \mathrm{cm}$ are achieved by convenient dc voltages of about $50 \mathrm{~V}$ applied to the MSM structure, which eliminates the need for pulsed high power voltage supplies. This approach provides a broadband high-power $\mathrm{THz}$ emitter.

A schematic of the THz emitter is shown in Fig. 1. Two interdigitated finger electrodes are processed by optical lithography on the surface of a semi-insulating GaAs wafer. The finger electrode width and spacing are $5 \mu \mathrm{m}$. Semiinsulating GaAs is an appropriate substrate because of its high carrier mobility and the high breakdown field. The electrode metallization consists of $5 \mathrm{~nm} \mathrm{Cr}$ and $200 \mathrm{~nm} \mathrm{Au}$. An opaque further metallization of $\mathrm{Cr}-\mathrm{Au}$ covers every second finger electrode spacing. The second metallization is isolated from the first by a polyimide layer (PMDA-ODA, Kapton) of about $2 \mu \mathrm{m}$ or a sputtered $\mathrm{SiO}_{x}$ layer of $560 \mathrm{~nm}$ thickness. When the finger electrodes are biased, the electric-field direction is reversed between successive fingers. Owing to the

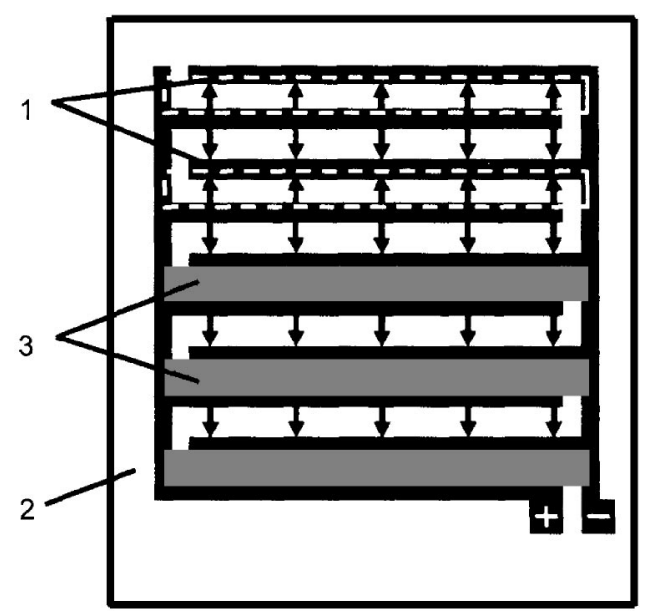

FIG. 1. Schematic sketch of the THz emitting MSM structure; (1) interdigitated finger electrodes, (2) SI GaAs substrate, (3) opaque metallization shadowing one electric field direction. The electric field direction is indicated by arrows. 
opaque second gold metallization on top of the finger electrodes optical excitation takes place only in substrate areas which exhibit the same field direction. Under optical excitation charge carriers are accelerated unidirectionally over the whole excited area. The $\mathrm{THz}$ radiation emitted through the substrate interferes constructively in the far field. This technique follows a similar approach of Yoneda et al. ${ }^{10}$ They use a diamond based interdigitated electrode structure excited with a high-power $\mathrm{Kr}^{*} \mathrm{~F}$ laser. The excitation in uniform field regions is achieved by the spatial modulation of the illuminating laser amplitude by a binary mask.

The setup for the detection of the $\mathrm{THz}$ waves is based on conventional electro-optical sensing. ${ }^{11,12}$ A mode-locked Ti:sapphire oscillator generates $50 \mathrm{fs}$ pulses at a wavelength of $800 \mathrm{~nm}$ and a repetition rate of $78 \mathrm{MHz}$. This beam is used to pump the room temperature operated $\mathrm{THz}$ emitter under normal incidence. The charge carriers are excited with an average power of max. $200 \mathrm{~mW}$ focused on a spot of about $80 \mu \mathrm{m}$. This corresponds to an excitation density of 1.7 $\times 10^{18} / \mathrm{cm}^{3}$. It should be noted that in the present device less than $20 \%$ of the incident radiation is absorbed in the PC due to $75 \%$ coverage of the surface with metallization and the reflectivity of the PC. This value can be increased by using an asymmetric MSM finger structure minimizing the shadowed region between the electrodes and an antireflection coating. The $\mathrm{THz}$ emitter is biased with a $50 \mathrm{kHz}$ square wave voltage. The $\mathrm{THz}$ wave trains emitted through the substrate are focused with off-axis parabolic mirrors on the detection system. A small fraction of the pump beam is split off, passed through an optical delay stage and realigned with the $\mathrm{THz}$ waves by a tin doped indium oxide (ITO) coated mirror. ${ }^{13}$ A $230 \mu \mathrm{m}$ thick ZnTe (110) crystal is used for the electro-optical detection of the $\mathrm{THz}$ field amplitude. The $\mathrm{THz}$ field induced change of the probe beam polarization is measured with a polarizing beam splitter and two photodiodes. The difference signal of the photodiodes is recorded by a lock-in amplifier locked to the modulation frequency of the $\mathrm{THz}$ emitter bias. The emitter design allows for modulation frequencies up to some MHz. The modulation frequency chosen here is well above from the amplitude noise of the pump laser. The duty cycle of the bias voltage was varied between $3.5 \%$ and $50 \%$.

Figure 2(a) shows the THz electric fields emitted at different acceleration fields. They are compared to the emission of a device without the second metallization layer biased at $20 \mathrm{kV} / \mathrm{cm}$. The THz field value was calculated from the relative intensity change of the probe beam at the detectors as described by Planken et al. ${ }^{14}$

The THz field amplitude increases linearly with rising acceleration field as shown in the inset of Fig. 2. This increase is based on the faster carrier acceleration in higher electric fields. At a bias-voltage duty cycle of $15 \%$ the slope of the $\mathrm{THz}$ amplitude versus the acceleration field becomes sublinear above $80 \mathrm{kV} / \mathrm{cm}$. This sublinearity vanishes using $3.5 \%$ duty cycle of the acceleration field. Higher duty cycles increase the substrate temperature because of the higher average power dissipation by the photocurrent and the associated reduction of the mobility. A maximum $\mathrm{THz}$ field of $85 \mathrm{~V} / \mathrm{cm}$ is reached at an acceleration field of $130 \mathrm{kV} / \mathrm{cm}$ with a duty cycle of $3.5 \%$. This THz field amplitude is comparable to the value achieved by Zhao et al. ${ }^{8}$ using a cooled semilarge aperture emitter operated at $400 \mathrm{~V}$. However, the
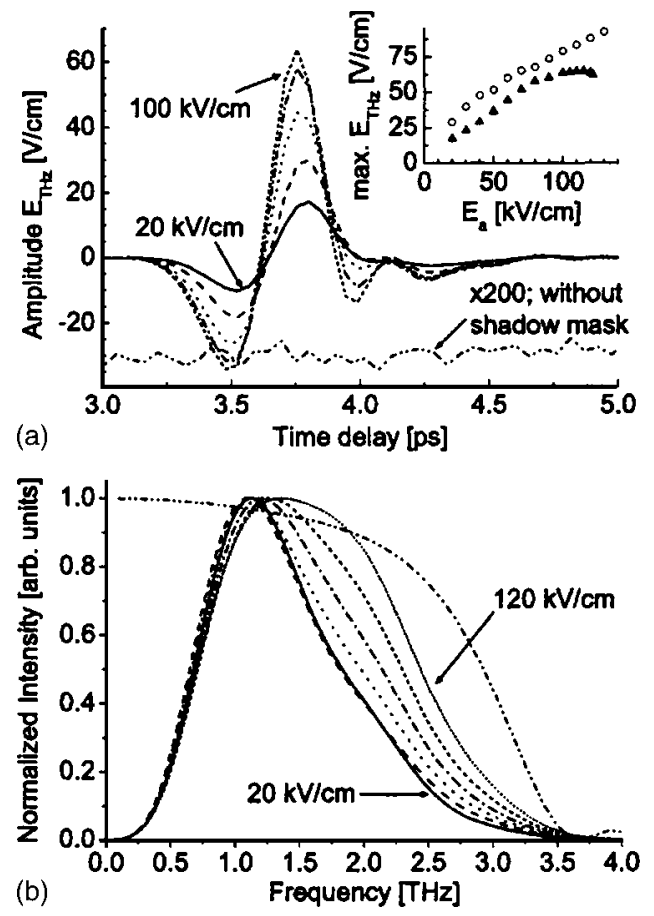

FIG. 2. (a) Time domain $\mathrm{THz}$ waveform for different acceleration fields of $20 \mathrm{kV} / \mathrm{cm}$ (solid line), $40 \mathrm{kV} / \mathrm{cm}$ (dashed line), $60 \mathrm{kV} / \mathrm{cm}$ (dotted line), $80 \mathrm{kV} / \mathrm{cm}$ (dashed-dotted line), and $100 \mathrm{kV} / \mathrm{cm}$ (short dashed line) at a duty cycle of $15 \%$ and an excitation density of $8.6 \times 10^{17} / \mathrm{cm}^{3}$. The dashed double-dotted line represents the (essentially vanishing) $\mathrm{THz}$ emission of a device without the second metallization at $20 \mathrm{kV} / \mathrm{cm}$ and $50 \%$ duty cycle, magnified by a factor of 200 and shifted vertically. The inset shows the maximum absolute $\mathrm{THz}$ field at different acceleration fields with $3.5 \%$ (circles) and $15 \%$ (triangles) duty cycle. (b) Power spectra calculated from the time domain $\mathrm{THz}$ electric field of (a), normalized to unity. The additional short dotted line corresponds to an acceleration field of $120 \mathrm{kV} / \mathrm{cm}$ and the dashed double dotted line represents the calculated response of the $\mathrm{ZnTe}$ analyzer crystal.

maximum signal to noise ratio is provided by a duty cycle of $50 \%$.

The dashed double dotted line in Fig. 2(a) represents the $\mathrm{THz}$ emission of a device without the second metallization at an acceleration field of $20 \mathrm{kV} / \mathrm{cm}$, magnified by a factor of 200 and shifted vertically. No THz field amplitude could be measured there. This is expected due to the alternating direction of the photocurrent within the alternating electric field between the electrodes. Hence in the far field the interference of the $\mathrm{THz}$ radiation is destructive.

To explain the observed waveforms and their field dependence the photocarrier dynamics have to be considered. ${ }^{5,6,15,16}$ In the limit of a dipole radiation approximation, the field amplitude of the emitted radiation in the far field is proportional to the time derivative of the transient photocurrent. The main cycle sets in with a small negative peak followed by a higher positive peak continued with a few smaller cycles. The main positive peak corresponds to the strong onset of the photocurrent due to the acceleration of carriers in the bias field of the electrodes. The subsequent negative peak is attributed to a decrease of the photocurrent by carrier deceleration. This peak increases relative to the main peak with increasing acceleration field owing to faster scattering into low mobility conduction band side valleys. At high excitation densities this negative peak is influenced by carrier-carrier scattering and screening of the acceleration field as discussed below. The preceding negative peak before 

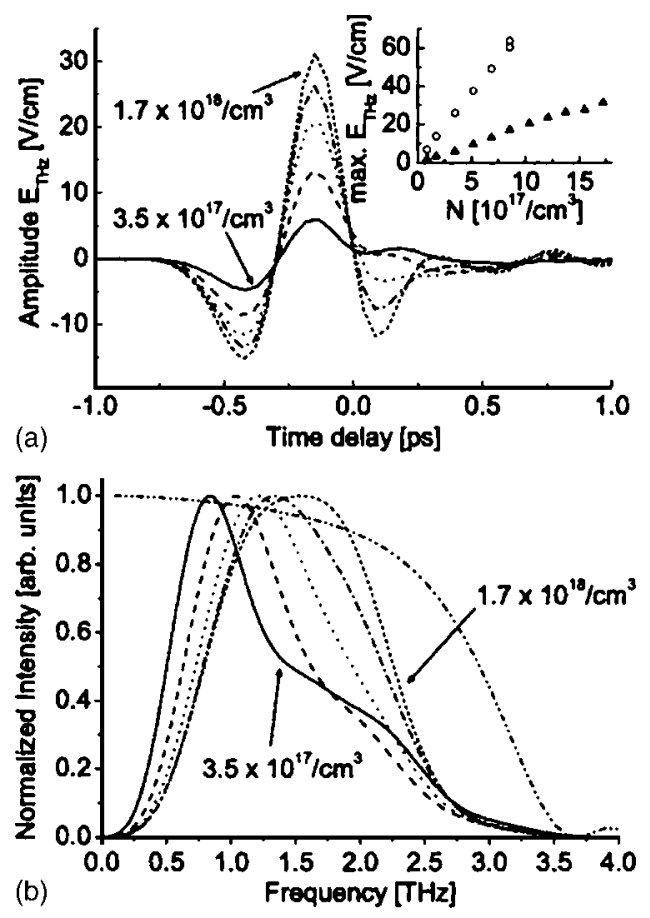

FIG. 3. (a) Normalized time domain THz waveform at $E_{a}=20 \mathrm{kV} / \mathrm{cm}$ and a duty cycle of $15 \%$ for different excitation densities of $3.5 \times 10^{17} / \mathrm{cm}^{3}$ (solid line), $6.9 \times 10^{17} / \mathrm{cm}^{3}$ (dashed line), $1.0 \times 10^{18} / \mathrm{cm}^{3}$ (dotted line), 1.4 $\times 10^{18} / \mathrm{cm}^{3}$ (dashed-dotted line), and $1.7 \times 10^{18} / \mathrm{cm}^{3}$ (short dashed line). The inset shows the maximum absolute $\mathrm{THz}$ field at different excitation densities for $E_{a}=20 \mathrm{kV} / \mathrm{cm}$ (triangles) and $120 \mathrm{kV} / \mathrm{cm}$ (circles). (b) Power spectra calculated from the time domain THz electric field of (a), normalized to unity. The dashed double dotted line shows the calculated response of the ZnTe analyzer crystal.

the main peak is assigned to pulse reshaping by the frequency dependent $\mathrm{THz}$ focus of the detection system. ${ }^{15,17}$

The normalized power spectra shown in Fig. 2(b) are calculated from the time domain data of Fig. 2(a) via a numeric Fourier transformation. These spectra extend from 0.5 to $3 \mathrm{THz}$. The high-frequency cut-off is detection limited owing to signal cancellation based on the difference of the phase velocity of the $\mathrm{THz}$ wave trains and the group velocity of the probe beam in the thick ZnTe crystal. The spectral peak frequency shifts from 1.1 to $1.5 \mathrm{THz}$ upon increasing the acceleration field from 20 to $120 \mathrm{kV} / \mathrm{cm}$ and is connected to the carrier dynamics discussed above. The highfrequency characteristics $(>3 \mathrm{THz})$ of the emitter has to be explored with a thinner detection crystal ${ }^{12}$ and shorter excitation and probe pulses.

In order to investigate the dependence of the $\mathrm{THz}$ emission on the excitation density we performed experiments at fixed acceleration fields. Figure 3(a) shows the THz amplitude recorded at $20 \mathrm{kV} / \mathrm{cm}$. The inset of Fig. 3(a) compares the maximum $\mathrm{THz}$ field amplitude at acceleration fields of $20 \mathrm{kV} / \mathrm{cm}$ and $120 \mathrm{kV} / \mathrm{cm}$. The THz field amplitude increases linearly in the excitation-density range investigated here due to the linear increase in photocurrent. The waveform exhibits a second negative peak which increases its amplitude with increasing excitation density relative to the main peak. This effect is attributed to stronger screening of the acceleration field, which provides a faster surge of the photocurrent at high carrier densities. ${ }^{15}$ This argumentation is supported by the corresponding spectra in the frequency domain. Figure 3(b) shows the frequency spectra calculated from the time domain data of (a). At low excitation densities the spectra show a narrow peak at about $750 \mathrm{GHz}$ followed by a broader shoulder. This shape is transformed to a broad characteristic at a center frequency of $1.6 \mathrm{THz}$ at high excitation densities.

We like to note the high potential to further increase the $\mathrm{THz}$ field of our device. Our concept is scalable towards larger emitting areas from presently approximately $5000 \mu \mathrm{m}^{2}$ to some $\mathrm{mm}^{2}$ and even $\mathrm{cm}^{2}$ which can be excited with amplified laser pulses. This in principle would enhance the emitted THz amplitude by orders of magnitude. Such a system could have comparable performance to optical rectification or difference frequency mixing ${ }^{2}$ and would open the pathway to nonlinear $\mathrm{THz}$ spectroscopy in many interesting systems. In addition such an emitter would be suitable for generation of continuous wave $\mathrm{THz}$ radiation by mixing two laser lines in a PC. ${ }^{18}$ This approach has often been limited by the power dissipation in a small excited area.

In conclusion, we have demonstrated the generation of high-intensity $\mathrm{THz}$ radiation based on a transient photocurrent in a large-area emitter with a novel electrode design in conjunction with selective shadowing of the optical excitation. This device overcomes disadvantages inherent to emitters using photoconductive antennas and large aperture emitters using two electrodes. In contrast to the latter the proposed device structure enables the exploitation of a large emitting area while keeping the bias field at large values. The high acceleration field which can be obtained in our concept makes the use of low-temperature grown GaAs favorable.

We acknowledge the preparation of the polyimide isolation layer by M. Guenther, and the support in sample preparation and helpful discussions by $\mathrm{H}$. Felsmann and $\mathrm{B}$. Schmidt.

${ }^{1}$ X.-C. Zhang, Y. Jin, and X. F. Ma, Appl. Phys. Lett. 61, 2764 (1992).

${ }^{2}$ K. Reimann, R. P. Smith, A. M. Weiner, T. Elsaesser, and M. Woerner, Opt. Lett. 28, 471 (2003).

${ }^{3}$ X.-C. Zhang and D. H. Auston, J. Appl. Phys. 71, 326 (1992).

${ }^{4}$ D. Grischkowsky, S. Keiding, M. V. Exter, and C. Fattinger, J. Opt. Soc. Am. B 7, 2006 (1990).

${ }^{5}$ For a review, see J. Shan and T. F. Heinz, in Ultrafast Dynamical Processes in Semiconductors, edited by K.-T. Tsen (Springer-Verlag, Berlin, 2004), pp. 1-56.

${ }^{6}$ L. Xu, X.-C. Zhang, D. H. Auston, and B. Jalali, Appl. Phys. Lett. 59, 3357 (1991).

${ }^{7}$ S. R. Andrews, A. Armitage, P. G. Huggard, and A. Hussain, Phys. Med. Biol. 47, 37053710 (2002).

${ }^{8}$ G. Zhao, R. N. Schouten, N. van der Valk, W. T. Wenckebach, and P. C. M. Planken, Rev. Sci. Instrum. 73, 1715 (2002).

${ }^{9}$ P. K. Benicewicz, J. P. Roberts, and A. J. Taylor, J. Opt. Soc. Am. B 11, 2533 (1994).

${ }^{10}$ H. Yoneda, K. Tokuyama, K. Ueda, H. Yamamoto, and K. Baba, Appl. Opt. 46, 6733 (2001).

${ }^{11}$ Q. Wu and X.-C. Zhang, Appl. Phys. Lett. 68, 1604 (1996).

${ }^{12}$ A. Leitenstorfer, S. Hunsche, J. Shah, M. C. Nuss, and W. H. Knox, Appl. Phys. Lett. 74, 1516 (1999).

${ }^{13}$ T. Bauer, J. S. Kolb, T. Löffler, E. Mohler, H. G. Roskos, and U. C. Pernisz, J. Appl. Phys. 92, 2210 (2002).

${ }^{14}$ P. C. M. Planken, H.-K. Nienhuys, H. J. Bakker, and T. Wenckebach, J. Opt. Soc. Am. B 18, 313 (2001).

${ }^{15}$ P. U. Jepsen, R. H. Jacobsen, and S. R. Keiding, J. Opt. Soc. Am. B 13, 2424 (1996).

${ }^{16}$ T.-A. Liu, M. Tani, and C.-L. Pan, J. Appl. Phys. 93, 2996 (2003).

${ }^{17}$ C. Fattinger and D. Grischkowsky, Appl. Phys. Lett. 54, 490 (1989).

${ }^{18}$ E. R. Brown, K. A. McIntosh, K. B. Nichols, and C. L. Dennis, Appl. Phys. Lett. 66, 285 (1995). 